\title{
Volume of fluid consumption during preparation for colonoscopy is possibly the single most important determinant of bowel preparation adequacy
}

\author{
Yuri Gorelika, Eisa Hag ${ }^{b}$, Tomer Hananyac, Ronit Leibad, Yehuda Chowers ${ }^{b, c}$, Elizabeth E. Half ${ }^{b, c}$ \\ Rambam Health Care Campus; Technion-Israel Institute of Technology, Haifa, Israel
}

Abstract

Background The effectiveness and safety of colonoscopy are directly dependent on the quality of bowel preparation. Multiple risk factors for inadequate bowel preparation (IBP) have been identified; however, IBP is still reported in $20-30 \%$ of cases in most studies. We aimed to identify modifiable predictors of the adequacy of bowel preparation using sodium picosulfate, and to recommend easily modifiable parameters to increase the success rate of colonoscopies.

\begin{abstract}
Methods This was a single-center observational study of adult outpatients referred for an elective colonoscopy. Patients were interviewed prior to colonoscopy; volume of liquids consumed was calculated as number of $200-\mathrm{mL}$ cups showed to the patient. Additional information, including medical history, diagnoses and regular medications, was procured from patients' medical records. Univariate and multivariate regression analyses were performed to identify factors significantly associated with IBP in a subgroup analysis of high-risk patients.
\end{abstract}

Results The rate of IBP in 1172 subjects was 19.4\%. This rate decreased as fluid consumption increased, with a further drop associated with shorter intervals from end of preparation to colonoscopy. Drinking < $1.4 \mathrm{~L}$ significantly increased the risk of IBP (odds ratio [OR] 3.62, 95\% confidence interval [CI] 2.65-4.95), while drinking $\geq 2 \mathrm{~L}$ was associated with adequate preparation (OR 0.09, 95\%CI 0-0.42). These associations were stronger in high-risk individuals.

Conclusions Greater fluid intake and short interval to colonoscopy are easily modifiable parameters that can substantially reduce the rate of IBP, especially among high-risk individuals.

Keywords Inadequate bowel preparation, colonoscopy, bowel cleansing, sodium picosulfate

Ann Gastroenterol 2021; 34 (1): 1-8

\section{Introduction}

The efficacy and safety of colonoscopy is highly dependent on the adequacy of bowel preparation [1-3]. Inadequate bowel

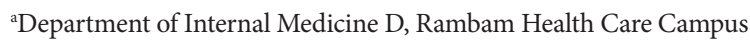
(Yuri Gorelik); b Gastroenterology Institute, Rambam Health Care Campus (Eisa Hag, Yehuda Chowers, Elizabeth E. Half); ${ }^{\mathrm{C}}$ uth and Bruce Rappaport Faculty of Medicine, Technion-Israel Institute of Technology (Tomer Hananya, Yehuda Chowers, Elizabeth E. Half); ${ }^{\mathrm{d} D e p a r t m e n t ~ o f ~}$ Epidemiology, Rambam Health Care Campus (Ronit Leiba), Haifa, Israel

Conflict of Interest: Elizabeth Half is a recipient of a research grant from Ferring Pharmaceuticals. All other authors report no financial or other conflicts of interest

Correspondence to: Elizabeth Half, MD, Department of Gastroenterology, Rambam Health Care Campus, Haifa, 3109601

Israel, e-mail: e_half@rambam.health.gov.il

Received 25 October 2020; accepted 29 December 2020; published online 14 June 2021

DOI: https://doi.org/10.20524/aog.2021.0642 preparation (IBP) is associated with lower detection rates of both benign and malignant lesions, longer procedure times and a more frequent need for a repeat procedure, with a resulting loss of work days, additional colonoscopic procedural risk to the patient and greater cost [3-6]. The rate of IBP varies among multiple studies, ranging from $5-67 \%$. A recently published, large-scale metaanalysis showed a calculated IBP rate of $19.9 \%$ [7]; therefore, improvement in preparation adequacy rate is crucial.

Previous studies have identified multiple risk factors associated with IBP [8-12]: e.g. sex and older age are weak predictors of IBP. Diabetes and underlying neurologic conditions-mainly dementia, stroke and Parkinson's disease-were consistently shown to be strong predictors of IBP in large-scale studies $[9,10]$. Various medications are associated with a higher risk of IBP, with opioids and tricyclic antidepressants being the strongest and most consistent in large-scale studies $[10,11,13]$. Factors associated with the preparation protocol (especially the effectiveness of split dose preparation and adding bisacodyl or senna to the standard preparation) were assessed in other studies, and were found 
to be mostly associated with better rates of adequate bowel preparation [14-17]. It has also been suggested that a delay between the end of preparation and the colonoscopy confers a higher risk for IBP [18-20]. However, most of the factors suggested by these studies are currently not modifiable, with the exception of shortening the time interval to the procedure (not always possible due to time of day) and splitting the dose of the medication chosen for preparation. In the current study, we sought to prospectively collect multiple patient characteristics, including modifiable factors associated with bowel preparation that have not been previously reported but could potentially be used to reduce IBP and improve the quality of colonoscopy.

\section{Patients and methods}

\section{Design}

We performed a prospective, single-center, observational study of adult outpatients undergoing colonoscopy, regardless of indication. The study took place between September 2016 and April 2018, at the Department of Gastroenterology, Rambam, Haifa, Israel. All subjects provided written informed consent. Included were adult patients referred for outpatient colonoscopy who had provided personal and medical information, as well as details concerning the bowel preparation procedure. Excluded were inpatients and patients who had undergone previous bariatric, small bowel, or colorectal surgical procedures. Patients with a previous history of other abdominal procedures, such as appendectomy or cholecystectomy, were included. The study protocol was approved in April 2016 by the Rambam institutional review board and conformed to the ethical guidelines of the 1975 Declaration of Helsinki.

\section{Data collection}

All demographic information, medical diagnoses, medications and indications for the procedure were extracted from patients' electronic medical records and physicians' referral letters. All patients were interviewed by a single nurse practitioner $(\mathrm{EH})$ regarding preparation-related information, using a standard questionnaire prior to colonoscopy. After the first 452 patients, the data were analyzed and a decision was made to extend the questionnaire to include all previous questions with the addition of the new variables listed.

Demographic and social information, medical and surgical history, as well as medications and lifestyle habits (e.g. tobacco use) were collected. Data regarding medical history were reviewed with the patients and recorded, including chronic constipation $(<3$ bowel movements per week), diarrhea ( $>3$ watery bowel movements per day), diabetes, inflammatory bowel disease, thyroid function disorder, rheumatic disease, heart disease, and neurological disorders such as previous cerebrovascular accident or Parkinson's disease. Subjects were queried as to when they had performed preparation steps, such as initiation of laxative sachets, as well as the time intervals between sachets and between laxative-initiated diarrheal episodes. Subjects were also interviewed regarding compliance with the bowel preparation protocol, including diet, bisacodyl use and the volume of liquids consumed defined by number of 200-mL volume cups presented during the interview. Patients who completed the interview were included in the final analysis, irrespective of factors such as measures of compliance.

\section{Bowel preparation}

The recommended bowel preparation protocol, including written instructions, was mailed to all patients scheduled for an outpatient colonoscopy at Rambam. The preparation protocol consisted of split-dose Picolax ${ }^{\oplus}$ (sodium picosulfate together with magnesium citrate). Each dose consisted of $16.1 \mathrm{~g}$ sodium picosulfate with magnesium citrate, which patients were instructed to dissolve in a cup of water. Patients scheduled for a morning procedure were instructed to stop all solid food at 12:00 the day before the procedure and drink only clear liquids until $3 \mathrm{~h}$ before the procedure; they were instructed to drink the first sachet at 14:00 and the second sachet at 20:00 the day before the procedure. Patients scheduled for an afternoon examination were instructed to discontinue solid food at 18:00 and consume the first sachet at 20:00 the day before the procedure, and the second sachet at 08:00 the day of the procedure. Patients were recommended to drink $1.6 \mathrm{~L}$ (8 cups) of clear liquids following each sachet.

All patients were recommended to take 2 tablets of bisacodyl 5 mg q.d., starting 3 days prior to colonoscopy. A low-fiber diet was recommended for 3 days prior to the procedure. All medications, except for antidiabetics, were permitted, and antiplatelets and anticoagulants were discontinued according to standard guidelines [21]. Standard dose aspirin (75-325 $\mathrm{mg}$ ) could be continued. During their interview, patients were shown a $200-\mathrm{mL}$ cup and asked to estimate how many such cups of water, or any other clear liquid, they had consumed after each sachet. The main endpoint was bowel preparation adequacy as assessed during colonoscopy.

Endoscopists with various levels of expertise, ranging from fellows to highly experienced senior endoscopists, performed the colonoscopies and evaluated bowel preparations. Colonoscopies were performed using Olympus 160 and 185 series and Fuji 600 series endoscopy systems.

The adequacy of bowel preparation was graded by the endoscopist according to the Boston Bowel Preparation Scale (BBPS), as previously described [22]. In summary, the BBPS is based on a 4-point scoring system applied separately to each of the 3 main segments of the colon [22]. The summation of the 3 "segment scores" gives a total BBPS score ranging from $0-9$. As previously recommended [21], bowel preparation was considered "good" if the BBPS was $\geq 6$, and $\geq 2$ in each colonic segment, or "inadequate" otherwise. A written explanation of the Boston scale was provided to the endoscopist upon request via the electronic endoscopic report.

\section{Statistical analysis}

The statistical analysis was performed using $\mathrm{R}$ version 3.4.0 (The R Foundation for Statistical Computing, Vienna, 
Austria). All clinical variables were compared between the adequately and inadequately prepared subjects. Continuous variables were presented as medians with interquartile ranges, and then compared using a Mann-Whitney $U$ test or Student's $t$-test, as appropriate. Dichotomous and categorical variables were presented as numbers, percentages and odds ratios (OR), then compared using Fisher's exact test and univariate logistic regression analysis, respectively, with the most frequent factor used as reference for categorical variables. Some continuous variables, such as the time interval from the end of preparation to the initiation of colonoscopy and any fluid consumption, were also analyzed as dichotomous or categorical variables for representative constructs (e.g. $<8$ or $>8 \mathrm{~h}$ from end of preparation to initiation of colonoscopy). Multivariate regression analysis was performed for variables that showed a significant difference between the adequate and inadequate preparation groups. To account for variability in preparation adequacy between endoscopists, an additional multivariate analysis was performed with the previously found predictors along with the performing endoscopist.

\section{Results}

Of the 1172 subjects who participated, 452 answered the original questionnaire and 720 the extended questionnaire; 619 $(52.8 \%)$ were males, and the mean age was 55.8 years. Baseline demographic characteristics of the subjects are presented in Table 1. Twenty-four endoscopists performed the examinations, with a median number of 33 colonoscopies per endoscopist.

IBP was found in 227 (19.4\%) subjects. A comparison of the demographic information, medical history and medications in the adequately and inadequately prepared subject groups, along with the ORs, is presented in Table 2. Typical colonoscopy images of adequately and inadequately prepared bowel segments seen during the study are presented in Fig. 1. In the univariate analysis, subjects with IBP were significantly older (mean age $60 \mathrm{vs.} 58$ years in the inadequately and adequately prepared groups, respectively; $\mathrm{P}=0.013)$ and weighed more than adequately prepared subjects ( 80 vs. $76 \mathrm{~kg}$, respectively; $\mathrm{P}<0.001)$. Diabetes $(35.2 \%$ of inadequately prepared vs. $19.4 \%$ of adequately prepared subjects, OR $2.26,95 \%$ confidence interval [CI] 1.6-3.14; $\mathrm{P}<0.001)$, history of psychiatric disease ( $11 \%$ of the IBP vs. $5.6 \%$ of adequately prepared subjects, OR 2.08, 95\%CI 1.21-3.5; $\mathrm{P}=0.007$ ), and heart disease (22\% of the IBP vs. $14.2 \%$ of adequately prepared subjects, OR $1.71,95 \% \mathrm{CI}$ 1.16-2.49; $\mathrm{P}=0.006$ ) were shown to be significantly associated with IBP. In addition, the use of several medication groups was also significantly associated with a higher risk of IBP. These included proton pump inhibitors, anti-hypertensives, statins, and diuretics, among others (Table 2).

A similar comparison of adequately and inadequately prepared patients for variables subsequently added to the second questionnaire $(n=720)$ is presented in Table 3. Education level significantly affected the risk of IBP; subjects with $<12$ years of education had a significantly higher risk for IBP (OR 3.06, 95\%CI 1.88-4.33) than those with academic education (bachelor's or an associate degree). In addition to weight, included in
Table 1 Baseline characteristics of the entire patient cohort $(n=1172)$

\begin{tabular}{lc} 
Variable & Value (\%) \\
\hline Female, $\mathrm{n}(\%)$ & $533(47.2)$ \\
Age, median (IQR), years & $58(20)$ \\
Weight, median (IQR), kg & $77(23.2)$ \\
Comorbidities, n (\%) & \\
Previous abdominal surgery & $480(40.9)$ \\
Heart failure & $184(15.6)$ \\
Diabetes & $223(22.4)$ \\
Psychiatric disease & $78(6.6)$ \\
IBD & $144(12.3)$ \\
Medications, $\mathrm{n}(\%)$ & \\
PPI & $166(14.2)$ \\
Antihypertensives & $500(42.7)$ \\
Statins & $457(39)$ \\
Antidepressants & $49(4.2)$ \\
Antipsychotics & $85(7.2)$ \\
Diuretics & $58(4.9)$ \\
Iron supplements & $75(6.4)$ \\
Opioids & $13(1.1)$ \\
\hline
\end{tabular}

$\overline{I Q R}$, interquartile range; IBD, inflammatory bowel disease; PPI, proton pump inhibitors

the original cohort, body mass index (BMI) was added to the extended questionnaires; as with weight, obesity (BMI $\geq 30 \mathrm{~kg} /$ $\mathrm{m}^{2}$ ) conferred a higher risk for IBP (OR 1.84, 95\%CI 1.2-2.78). A subgroup analysis of various antidiabetic medications in diabetic patients did not reveal significant differences in the risk of IBP for any of the medications, including insulin. The use of 5 or more medication groups, not limited to those specifically mentioned above, was considered as polypharmacy and was associated with a significantly higher risk of IBP (OR 2.49, 95\%CI 1.65-3.75). These associations were not found in the multivariate analysis.

Multiple parameters of the bowel preparation process were analyzed. A comparison of these variables between adequately and inadequately prepared patients is shown in Table 4. Adequate cleaning of the right colon (BBPS $\geq 2$ ) was recorded in all adequately prepared patients and in only $42 / 227$ (18.5\%) of the inadequately prepared patients.

An interval of more than $8 \mathrm{~h}$ from the end of preparation to the beginning of the examination conferred a significantly higher risk for IBP, irrespectively of the time of the procedure $(54.6 \%$ of the inadequately prepared vs. $41.3 \%$ of adequately prepared subjects, OR 1.71, 95\%CI 1.27-2.32; $\mathrm{P}<0.001$ ). In addition, higher rates of IBP were seen as the time from end of preparation to colonoscopy increased (Fig. 2A). An analysis comparing the volume of fluids consumed during preparation and adequacy showed that compared to subjects who drank 1.4L-2.0L of clear liquids subjects who drank $<1.4 \mathrm{~L}$ after each sachet had a significantly higher risk of IBP (OR 3.62, 95\%CI 2.65-4.95). Subjects who drank $>2 \mathrm{~L}$ of liquids were substantially more likely to have a successful preparation (OR 0.09, 95\%CI 0-0.42). 
Table 2 Patient demographic and clinical variables. Univariate comparison between adequate and inadequate preparation

\begin{tabular}{|c|c|c|c|c|}
\hline Variable & $\begin{array}{l}\text { Adequately prepared } \\
(\mathrm{n}=945,80.6 \%)\end{array}$ & $\begin{array}{l}\text { Inadequately prepared } \\
(\mathrm{n}=227,19.4 \%)\end{array}$ & P-value & $\begin{array}{l}\text { OR for inadequate } \\
\text { preparation }(95 \% \mathrm{CI})\end{array}$ \\
\hline Age (years) & $58(21)$ & $60(18)$ & $0.01^{*}$ & \\
\hline Female, n (\%) & $459(48.6 \%)$ & $94(41.4 \%)$ & 0.05 & $0.75(0.55-1.01)$ \\
\hline Weight (kg) & $76(22)$ & $80(22)$ & $<0.01$ & \\
\hline \multicolumn{5}{|l|}{ Comorbidities, n (\%) } \\
\hline Abdominal surgery (not including bowel procedures) & $387(41)$ & $93(41)$ & $>0.99$ & $1(0.74-1.36)$ \\
\hline Diabetes & $183(19.4)$ & $80(35.2)$ & $<0.01$ & $2.26(1.63-3.14)$ \\
\hline Heart disease & $134(14.2)$ & $50(22)$ & 0.01 & $1.71(1.16-2.49)^{*}$ \\
\hline Chronic kidney disease & $34(3.6)$ & $13(5.7)$ & 0.19 & $1.63(0.77-3.23)$ \\
\hline Neurologic disorder & $55(5.8)$ & $19(8.4)$ & 0.17 & $1.48(0.81-2.59)$ \\
\hline Rheumatic disease & $56(5.9)$ & $13(5.7)$ & $>0.99$ & $0.96(0.47-1.83)$ \\
\hline IBD & $119(12.6)$ & $25(11)$ & 0.57 & $0.86(0.52-1.37)$ \\
\hline Psychiatric disease & $53(5.6)$ & $25(11)$ & 0.01 & $2.08(1.21-3.5)$ \\
\hline Hypothyroidism & $103(10.9)$ & $23(10.1)$ & 0.81 & $0.92(0.55-1.5)$ \\
\hline \multicolumn{5}{|l|}{ Medications, n (\%) } \\
\hline Proton pump inhibitors & $117(12.4)$ & 49 (21.6) & $<0.01$ & $1.95(1.31-2.86)$ \\
\hline Antidepressants & $40(4.2)$ & $19(8.4)$ & 0.02 & $2.07(1.11-3.74)^{*}$ \\
\hline Antipsychotics & $61(6.5)$ & $24(10.6)$ & 0.05 & $1.71(1.00-2.87)^{*}$ \\
\hline Diuretics & $37(3.9)$ & $21(9.3)$ & $<0.01$ & $2.5(1.36-4.49)^{*}$ \\
\hline Iron supplementation & $55(5.8)$ & $20(8.8)$ & 0.13 & $1.56(0.87-2.72)$ \\
\hline Opioids & $7(0.7)$ & $6(2.6)$ & 0.03 & $3.63(1.00-12.76)^{*}$ \\
\hline Antihypertensives & $377(39.9)$ & $123(54.2)$ & $<0.01$ & $1.78(1.32-2.41)^{*}$ \\
\hline Statins & $342(36.2)$ & $115(50.7)$ & $<0.01$ & $1.81(1.34-2.45)^{*}$ \\
\hline Thyroid hormone replacement & $79(8.4)$ & $22(9.7)$ & 0.15 & $1.18(0.68-1.96)$ \\
\hline
\end{tabular}

${ }^{*}$ Non-significant when adjusted in multivariate analysis for weight, diabetes and fluid consumption

OR, odds ratio; CI, confidence interval; IBD, inflammatory bowel disease
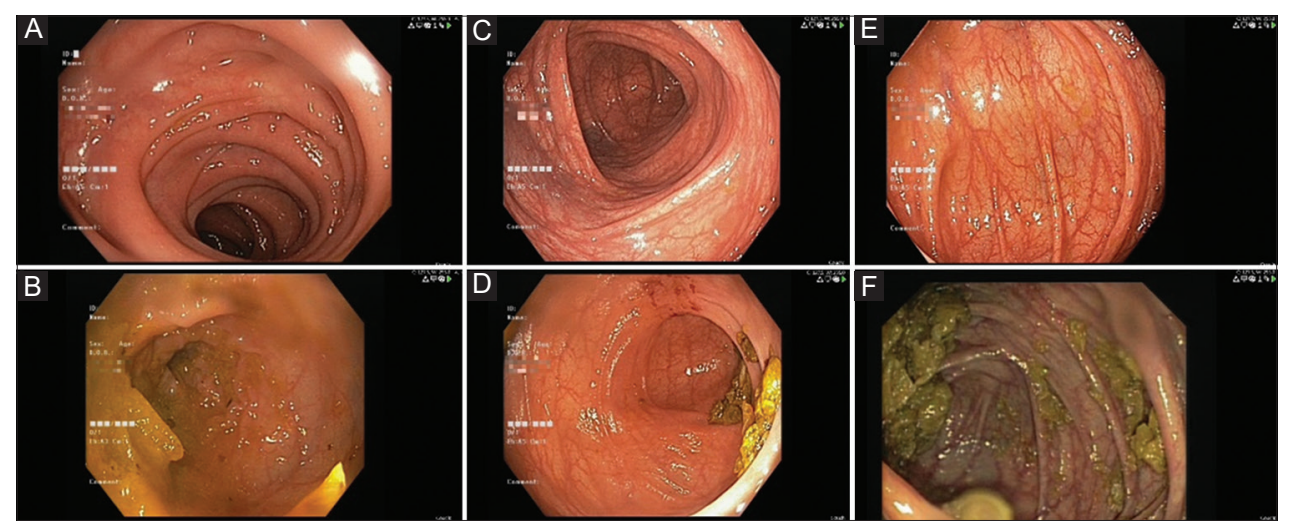

Figure 1 Typical colonoscopy images of adequately and inadequately prepared bowel segments seen during the study. Images taken in the Rambam healthcare campus endoscopy suite. Images include adequately and inadequately prepared left colon (A and B respectively); adequately and inadequately prepared transverse colon ( $\mathrm{C}$ and $\mathrm{D}$ respectively); and, adequately and inadequately prepared left colon (E and F respectively)

Moreover, out of 66 subjects who drank $>2$ L of fluids after each sachet, only one had inadequate preparation. A logistic regression of the rate of IBP plotted against volume of liquids consumed within a short time interval to colonoscopy, compared to all other patients, is shown in Fig. 2B. The IBP rate dropped consistently as the volume of liquids consumed increased, with a further drop of up to an approximate $50 \%$ for subjects who also had a short interval between end of preparation and colonoscopy. 
Table 3 Summary and comparison between patients with adequate and inadequate preparation. Demographic and clinical variables collected in the second (extended) questionnaire only

\begin{tabular}{|c|c|c|c|c|}
\hline Variable & $\begin{array}{l}\text { Adequately prepared } \\
\qquad(\mathrm{n}=579,80.4 \%)\end{array}$ & $\begin{array}{c}\text { Inadequately prepared } \\
\quad(\mathrm{n}=141,19.6 \%)\end{array}$ & P-value & $\begin{array}{l}\text { OR for inadequate } \\
\text { preparation }(95 \% \mathrm{CI})\end{array}$ \\
\hline Height, median (IQR), cm & $168(13)$ & $170(13)$ & $0.03^{*}$ & \\
\hline BMI, median (IQR), $\mathrm{kg} / \mathrm{m}^{2}$ & $26.3(5.9)$ & $27.5(6.7)$ & $<0.01$ & \\
\hline Obese $\left(\mathrm{BMI} \geq 30 \mathrm{~kg} / \mathrm{m}^{2}\right): \mathrm{n}(\%)$ & $127(21.9)$ & $48(34)$ & $<0.01$ & $1.84(1.2-2.78)^{\star}$ \\
\hline Smoking: $\mathrm{n}(\%)$ & $92(15.9)$ & $30(21.3)$ & 0.13 & $1.43(0.87-2.31)$ \\
\hline Pack-years & $15(20.7)$ & $15(21.8)$ & 0.6 & $0.79(0.53-1.17)$ \\
\hline Regular exercise: $\mathrm{n}(\%)$ & $216(37.3)$ & $43(30.5)$ & 0.14 & $0.74(0.48-1.11)$ \\
\hline Carbonated drinks ( $\geq 2$ per week): $\mathrm{n}(\%)$ & $154(26.6)$ & $51(36.2)$ & 0.03 & $1.56(1.03-2.35)^{*}$ \\
\hline \multicolumn{5}{|l|}{ Marital status } \\
\hline Married & $450(77.7)$ & $107(75.9)$ & & Reference \\
\hline Single & $86(14.9)$ & $13(9.2)$ & & $0.64(0.33-1.14)$ \\
\hline Widower & $24(4.1)$ & $11(7.8)$ & & $1.93(0.88-3.97)$ \\
\hline Divorcee & $19(3.3)$ & $10(7.1)$ & & $2.21(0.96-4.8)$ \\
\hline \multicolumn{5}{|l|}{ Education level, n (\%) } \\
\hline Bachelor's or associate degree & $392(67.7)$ & $65(46)$ & & Reference \\
\hline Less than 12 years & $71(12.2)$ & $36(25.5)$ & & $3.06(1.88-4.33)$ \\
\hline 12 years & $116(20)$ & $40(28)$ & & $2.08(1.33-3.24)$ \\
\hline \multicolumn{5}{|l|}{ Bowel habits, n (\%) } \\
\hline Normal & $749(79.3)$ & $173(76.2)$ & & Reference \\
\hline Diarrhea & $81(8.5)$ & $17(7.5)$ & & $0.91(0.51-1.53)$ \\
\hline Constipation & $115(12.2)$ & $37(16.3)$ & & $1.39(0.92-2.07)$ \\
\hline \multicolumn{5}{|l|}{ Nationality, n (\%) } \\
\hline Ashkenazi Jew & $333(57.5)$ & $60(42.5)$ & & Reference $^{*}$ \\
\hline Sephardic Jew & $142(24.5)$ & $38(26.9)$ & & $1.48(0.94-2.32)$ \\
\hline Arab Muslim & $48(8.3)$ & $21(14.9)$ & & $2.43(1.34-4.3)$ \\
\hline Arab Christian & $38(6.6)$ & $12(8.5)$ & & $1.75(0.84-3.46)$ \\
\hline Druze & $18(3.1)$ & $10(7.1)$ & & $3.1(1.31-6.9)$ \\
\hline \multicolumn{5}{|l|}{ Comorbidities: $\mathrm{n}(\%)$} \\
\hline Pulmonary disease & $30(5.2)$ & $19(13.5)$ & $<0.01$ & $2.84(1.46-5.42)$ \\
\hline HbAlC $\dagger$, median (IQR), \% & $6.8(1.4)$ & $6.8(1.2)$ & 0.77 & \\
\hline \multicolumn{5}{|l|}{ Medications, n (\%) } \\
\hline Polypharmacy ( $\geq 5$ different medication groups) & $121(20.9)$ & $56(39.7)$ & $<0.01$ & $2.49(1.65-3.75)^{*}$ \\
\hline Insulin $^{+}$ & $34(5.9)$ & $19(13.5)$ & 0.47 & $1.31(0.61-2.76)$ \\
\hline Sulfonylurea $^{\dagger}$ & $16(13.6)$ & $2(3.8)$ & 0.06 & $0.26(0.03-1.16)$ \\
\hline Metformin $^{+}$ & $88(74.6)$ & $42(80.8)$ & 0.44 & $1.43(0.61-3.59)$ \\
\hline DPP4 inhibitors ${ }^{\dagger}$ & $26(22)$ & $9(17.3)$ & 0.54 & $0.74(0.28-1.81)$ \\
\hline
\end{tabular}

${ }^{*}$ Non-significant when adjusted in multivariate analysis for weight, education level, diabetes, and fluid consumption

${ }^{\dagger}$ Subgroup analysis of diabetic patients

OR, odds ratio; CI, confidence interval; IQR, interquartile range; BMI, body mass index; HbA1C, glycosylated hemoglobin; DPP4, dipeptidyl peptidase-4

Multivariate regression analysis that included multiple factors significantly associated with IBP on univariate analysis, along with the performing endoscopist, showed that only weight, presence of diabetes, psychiatric disease, volume of fluids consumed, and time interval to colonoscopy were significantly associated with an elevated risk for IBP in the entire cohort $(n=1172)$. ORs and $95 \%$ CIs of the multivariate regression analysis results are shown in Table 5. 
Table 4 Summary and comparison of bowel preparation related variable between patients with adequate and inadequate bowel preparation

\begin{tabular}{|c|c|c|c|c|}
\hline Variable & $\begin{array}{l}\text { Adequately prepared } \\
\qquad(\mathrm{n}=945,80.6 \%)\end{array}$ & $\begin{array}{l}\text { Inadequately prepared } \\
\qquad(\mathrm{n}=227,19.4 \%)\end{array}$ & P-value & $\begin{array}{l}\text { OR for inadequate } \\
\text { preparation }(95 \% \mathrm{CI})\end{array}$ \\
\hline Time between Picolax sachets (h) & $6(6)$ & $6(6)$ & 0.22 & \\
\hline Time between $1^{\text {st }}$ sachet and bowel movement $(\mathrm{h})$ & $2(2)$ & $2(2)$ & 0.11 & \\
\hline Time between $2^{\text {nd }}$ sachet and bowel movement (h) & $1(0.5)$ & $1(1.5)$ & 0.13 & \\
\hline Time between end of preparation to colonoscopy (h) & $6(7)$ & $9(7.5)$ & $<0.01$ & \\
\hline More than $8 \mathrm{~h}, \mathrm{n}(\%)$ & $390(41.3)$ & $124(54.6)$ & $<0.01$ & $1.71(1.27-2.32)$ \\
\hline Afternoon exam, n (\%) & $352(37.2)$ & $56(24.7)$ & $<0.01$ & $0.55(0.39-0.77)$ \\
\hline Bisacodyl use, $\mathrm{n}(\%)$ & $811(85.8)$ & $203(89.4)$ & 0.16 & $1.40(0.87-2.32)$ \\
\hline Total bisacodyl (tablets) & $4(3)$ & $4(3)$ & & 0.79 \\
\hline Volume of liquids after each sachet, & $1600(400)$ & $1500(500)$ & $<0.01$ & \\
\hline$<1.4 \mathrm{~L}, \mathrm{n}(\%)$ & $172(18.2)$ & $105(46.6)$ & $<0.01$ & $3.62(2.65-4.95)$ \\
\hline 1.4L-2.0 L, n (\%) & $706(74.9)$ & $119(52.9)$ & 0.02 & Reference \\
\hline$>2.0 \mathrm{~L}, \mathrm{n}(\%)$ & $65(6.9)$ & $1(0.4)$ & & $0.09(0-0.42)$ \\
\hline Bowel movements & $8(4)$ & $6(3)$ & & $<0.001$ \\
\hline BBPS, median (IQR) & $8(2)$ & $5(2)$ & & $<0.001$ \\
\hline
\end{tabular}

OR, odds ratio; $C I$, confidence interval; BBPS, Boston bowel preparation score; IQR, interquartile range
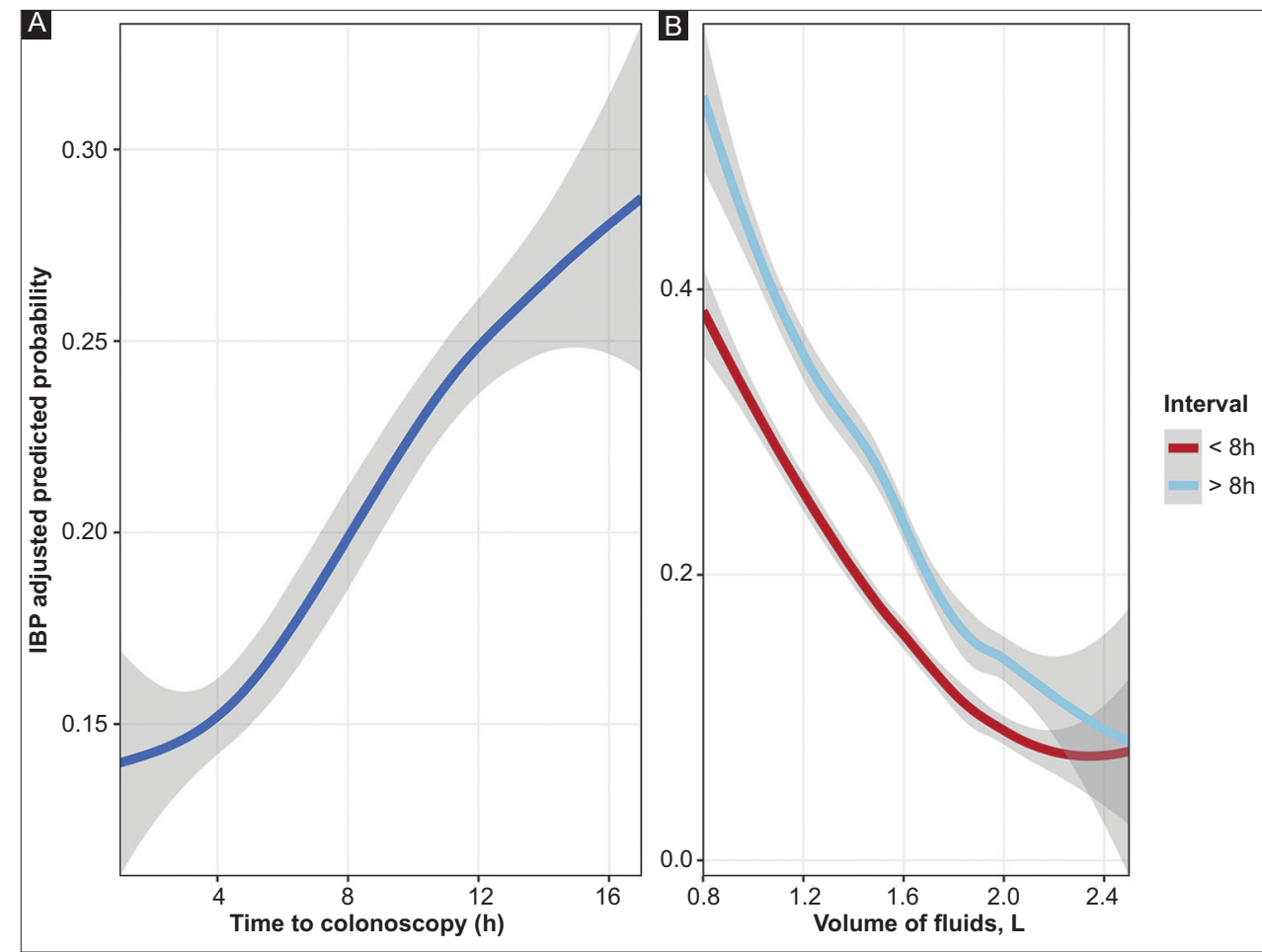

Figure 2 Predicted probability of inadequate bowel preparation (IBP) adjusted for variables significantly associated with IBP. A function of time (h) from end of preparation to colonoscopy (A) and volume of clear liquids consumed after each sachet of picosulfate, stratified by time to colonoscopy (B). Grey outlines represent the standard errors of the models

We further analyzed a subgroup of patients with favorable preparation features (drank $\geq 1.6 \mathrm{~L}$ of liquids and waited $\leq 8 \mathrm{~h}$ between end of preparation and beginning of examination). In this group, only $41 / 487$ (8.4\%) failed bowel preparation, compared to an IBP rate of $27 \%$ in the 685 patients who either drank $<1.6 \mathrm{~L}$ of fluid or had their examination delayed $(\mathrm{P}<0.001)$. A subgroup analysis of patients at high risk for IBP, such as diabetics or individuals weighing more than $90 \mathrm{~kg}$, revealed that among 96 diabetic individuals with both favorable features, inadequate preparation was seen in only 11 (11.5\%), while 69/167 
Table 5 Multivariate analysis of factors significantly associated with inadequate bowel preparation

\begin{tabular}{lc}
\hline Variable & Adjusted OR (95\%CI) \\
\hline Diabetes & $1.95(1.37-2.76)$ \\
Weight (odds ratio per 1 kg increase) & $1.01(1.005-1.02)$ \\
Psychiatric disease & $1.96(1.13-3.31)$ \\
$\begin{array}{l}\text { Volume of liquids (odds ratio per } \\
200 \text { mL increase) }\end{array}$ & $0.73(0.68-0.8)$ \\
Examination delay (>8 h) & $1.48(1.09-2.02)$ \\
\hline OR, odds ratio; CI, confidence interval &
\end{tabular}

(36.9\%) of diabetic patients who did not have both features failed preparation $(\mathrm{P}<0.001)$. Only 10/114 $(8.8 \%)$ subjects with both favorable features who weighed $90 \mathrm{~kg}$ or more failed preparation, in comparison to a failed preparation in 61/167 (36.5\%) subjects with this weight but without both favorable parameters $(\mathrm{P}<0.001)$.

\section{Discussion}

Adequate bowel preparation is essential for ideal endoscopic diagnostic yield. In this study, we prospectively studied 1172 patients in order to: 1) identify features associated with a high risk for IBP; 2) compare these findings to previous reports; and 3) search for bowel preparation features that can easily be modified and implemented into bowel preparation protocols to potentially lower the rate of IBP, particularly in high-risk individuals.

The rate of IBP in our cohort was $19.4 \%$, similar to the IBP rates found in other studies, including a recent large metaanalysis performed by Mahmood et al [7,21]. The adequacy of bowel preparation, graded according to the BBPS, was also similar to other studies where preparation was mostly with sodium picosulfate $[23,24]$. Multivariate adjustment revealed unmodifiable variables (e.g. obesity, diabetes, and education level) significantly associated with IBP, all of which are well-established risk factors in the literature $[7,8]$. Interestingly, and consistently with previous findings, a higher BMI tended to raise the risk of IBP significantly, especially in males [8], though our study had a male predominance. Moreover, when females alone were analyzed, the effects of weight and BMI were attenuated (OR for obesity as a risk factor for IBP in females alone was $1.75,95 \% \mathrm{CI}$ 0.93-3.24). When multiple medication groups were assessed (e.g. opiates, anti-hypertensive drugs, and lipid lowering drugs) some were shown to be associated with IBP, similarly to previous reports $[7,8]$. Additionally, polypharmacy was associated with an increased risk of IBP, regardless of medication type (Table 3). While this association has been demonstrated previously [19], in our study this finding was not significantly associated with IBP after adjustment for multiple other factors (Tables 3,5).

Extensive bowel preparation characteristics were collected and analyzed, including volume of clear liquids consumed during colonoscopy preparation, which to the best of our knowledge has not been previously reported. We show that the volume of liquids consumed with each sachet was independently associated with the rate of IBP, where only $1 / 66$ patients who consumed $\geq 2 \mathrm{~L}$ after each sachet had inadequate preparation. Furthermore, this is the first study to show a direct and continuous association between volume of fluids consumed and IBP rate. This continuous reduction in rate was seen as fluid consumption exceeded the instructions provided in the picosulfate leaflet, which recommends at least $1.25 \mathrm{~L}$ ( 5 cups) and only $750 \mathrm{~mL}$ (3 cups or $250 \mathrm{~mL}$ ) of clear liquids following the first and second sachets, respectively. In an effort to identify additional modifiable preparation-related features, we observed that the rate of IBP decreased further in a subgroup of patients whose colonoscopies were performed $<8 \mathrm{~h}$ after they took the second sachet of sodium picosulfate. This finding was independent of whether the procedure took place during morning or afternoon hours. IBP rates were low even in high-risk diabetic, obese, or older patients who met this criterion. A longer time interval between bowel preparation and colonoscopy has long been shown to be a risk factor for IBP $[7,8]$; however, the further improvement we found with greater liquid consumption has major significance. An important reservation regarding these findings is the possible risk of electrolyte disturbances with consumption of high volumes of water during bowel preparation. Our subjects were outpatients and we did not perform any blood sample analysis. Therefore, further research is needed, and balanced fluids should be considered when high-volume preparations are recommended or studied. However, we are unaware of any hospitalizations or deaths that occurred in our cohort.

Several limitations of our study need to be addressed. Data were collected through questionnaires filled out by the patient with the assistance of a nurse practitioner immediately prior to colonoscopy, and therefore patient recall might cause bias with regard to clinical as well as preparation-associated information. Moreover, it is likely that subjects would feel some degree of unease about admitting to poor compliance with the preparation protocol. The large number of patients assessed in this study may alleviate this potential bias. Furthermore, this study was conducted in a single tertiary center in Israel, which might affect the generalizability of the results. However, our cohort was demographically diverse and included a variety of ages, ethnicities and educational backgrounds, which may have mitigated the aforementioned drawback. Since all patients were scheduled for an elective examination, the indication for colonoscopy was not recorded, though this information could have helped identify subgroups that would benefit from high-volume fluid preparation. Another substantial limitation is the difficulty of achieving full adherence to guidelines and mainly the strong recommendation of initiation of colonoscopy within $5 \mathrm{~h}$ of consumption of the last dose of bowel preparation [21]. The difficulty, especially with early morning examinations, is a well-known challenge. The lack of post-procedure follow up for any adverse effects is an additional limitation. However, we are unaware of any preparationassociated post colonoscopy hospitalizations. Finally, since all subjects in the study used sodium picosulfate for preparation, results might not be generalizable to other preparation protocols and further investigations are required.

In conclusion, along with previously suggested risk factors for IBP, also found in the current study, herein we show 2 


\section{Summary Box}

\section{What is already known:}

- Inadequate bowel preparation (IBP) is associated with reduced visualization, missed lesions, and an increased risk of colonoscopy

- A large meta-analysis estimated the median rate of IBP to be $26 \%$

- Multiple risk factors for IBP have been identified, including age, comorbidities such as obesity and diabetes, use of specific medications, and a long interval between end of bowel preparation and colonoscopy

- Other than compliance with preparation protocols, there are few easily modifiable risk factors for IBP

\section{What the new findings are:}

- The volume of liquids consumed with each sachet was highly associated with the rates of IBP

- Increasing rates of IBP were observed in a continuous relation with longer time intervals between end of preparation and colonoscopy

- The combination of a larger volume of fluids consumed during the preparation, as well as a $<8$-h gap between the end of preparation and colonoscopy, additively reduced the rate of IBP

- Even in diabetic individuals at risk for preparation failure, low rates of IBP were observed in the group that consumed a large volume of fluids and had a short interval to colonoscopy

modifiable bowel preparation factors, low fluid intake and a long interval to colonoscopy, that can easily be incorporated into preparation protocols to improve bowel preparation with sodium picosulfate. Further prospective interventional studies will be necessary before definitive conclusions can be drawn.

\section{References}

1. Rex DK, Imperiale TF, Latinovich DR, Bratcher LL. Impact of bowel preparation on efficiency and cost of colonoscopy. Am J Gastroenterol 2002;97:1696-1700.

2. Froehlich F, Wietlisbach V, Gonvers JJ, Burnand B, Vader JP. Impact of colonic cleansing on quality and diagnostic yield of colonoscopy: the European Panel of Appropriateness of Gastrointestinal Endoscopy European multicenter study. Gastrointest Endosc 2005;61:378-384.

3. Lebwohl B, Kastrinos F, Glick M, Rosenbaum AJ, Wang T, Neugut AI. The impact of suboptimal bowel preparation on adenoma miss rates and the factors associated with early repeat colonoscopy. Gastrointest Endosc 2011;73:1207-1214.

4. Harewood GC, Sharma VK, de Garmo P. Impact of colonoscopy preparation quality on detection of suspected colonic neoplasia. Gastrointest Endosc 2003;58:76-79.

5. Hendry PO, Jenkins JT, Diament RH. The impact of poor bowel preparation on colonoscopy: a prospective single centre study of 10571 colonoscopies. Colorectal Dis 2007;9:745-748.

6. Chokshi RV, Hovis CE, Hollander T, Early DS, Wang JS. Prevalence of missed adenomas in patients with inadequate bowel preparation on screening colonoscopy. Gastrointest Endosc 2012;75:1197-1203.

7. Mahmood S, Farooqui SM, Madhoun MF. Predictors of inadequate bowel preparation for colonoscopy: a systematic review and metaanalysis. Eur J Gastroenterol Hepatol 2018;30:819-826.

8. Gandhi K, Tofani C, Sokach C, Patel D, Kastenberg D, Daskalakis C. Patient characteristics associated with quality of colonoscopy preparation: a systematic review and meta-analysis. Clin Gastroenterol Hepatol 2018;16:357-369.

9. Menees SB, Elliott E, Govani S, et al. The impact of bowel cleansing on follow-up recommendations in average-risk patients with a normal colonoscopy. Am J Gastroenterol 2014;109:148-154.

10. Yee R, Manoharan S, Hall C, Hayashi A. Optimizing bowel preparation for colonoscopy: what are the predictors of an inadequate preparation? Am J Surg 2015;209:787-792.

11. Dik VK, Moons LM, Hüyük M, et al; Colonoscopy Quality Initiative. Predicting inadequate bowel preparation for colonoscopy in participants receiving split-dose bowel preparation: development and validation of a prediction score. Gastrointest Endosc 2015;81:665-672.

12. Rotondano G, Rispo A, Bottiglieri ME, et al; SIED Campania PISCoPO study group investigators. Quality of bowel cleansing in hospitalized patients undergoing colonoscopy: A multicentre prospective regional study. Dig Liver Dis 2015;47:669-674.

13. Borg BB, Gupta NK, Zuckerman GR, Banerjee B, Gyawali CP. Impact of obesity on bowel preparation for colonoscopy. Clin Gastroenterol Hepatol 2009;7:670-675.

14. Kilgore TW, Abdinoor AA, Szary NM, et al. Bowel preparation with split-dose polyethylene glycol before colonoscopy: a meta-analysis of randomized controlled trials. Gastrointest Endosc 2011;73:1240-1245.

15. Vradelis S, Kalaitzakis E, Sharifi Y, et al. Addition of senna improves quality of colonoscopy preparation with magnesium citrate. World J Gastroenterol 2009;15:1759-1763.

16. Valiante F, Bellumat A, De Bona M, De Boni M. Bisacodyl plus split 2-L polyethylene glycol-citrate-simethicone improves quality of bowel preparation before screening colonoscopy. World $J$ Gastroenterol 2013;19:5493-5499.

17. Ibáñez M, Parra-Blanco A, Zaballa $P$, et al. Usefulness of an intensive bowel cleansing strategy for repeat colonoscopy after preparation failure. Dis Colon Rectum 2011;54:1578-1584.

18. Ness RM, Manam R, Hoen H, Chalasani N. Predictors of inadequate bowel preparation for colonoscopy. Am J Gastroenterol 2001;96:1797-1802.

19. Lebwohl B, Wang TC, Neugut AI. Socioeconomic and other predictors of colonoscopy preparation quality. Dig Dis Sci 2010;55:2014-2020.

20. Papastergiou V, Papasavvas S, Mathou N, et al. A delayed onset of bowel activity after the start of conventional polyethylene glycol predicts inadequate colon cleansing before colonoscopy: a prospective observational study. United European Gastroenterol J 2016;4:199-206.

21. Hassan C, East J, Radaelli F, et al. Bowel preparation for colonoscopy: European Society of Gastrointestinal Endoscopy (ESGE) Guideline - Update 2019. Endoscopy 2019;51:775-794.

22. Lai EJ, Calderwood AH, Doros G, Fix OK, Jacobson BC. The Boston bowel preparation scale: a valid and reliable instrument for colonoscopy-oriented research. Gastrointest Endosc 2009;69:620-625.

23. Manes G, Repici A, Hassan C; MAGIC-P study group. Randomized controlled trial comparing efficacy and acceptability of split- and standard-dose sodium picosulfate plus magnesium citrate for bowel cleansing prior to colonoscopy. Endoscopy 2014;46:662-669.

24. Manes G, Amato A, Arena M, et al. Efficacy and acceptability of sodium picosulphate/magnesium citrate versus low-volume PEGascorbic acid for colon cleansing: a randomized controlled trial. Colorectal Dis 2013;15:1145-1153. 